\title{
Measurement of Ultra Low Transverse Emittance at REGAE
}

\author{
M. Hachmann ${ }^{\mathrm{a}}$, K. Flöttmann ${ }^{\mathrm{a}}$ \\ ${ }^{a}$ Deutsches Elektronen-Synchrotron DESY, Notkestraße 85, 22607 Hamburg, Germany
}

\begin{abstract}
The linear accelerator REGAE at DESY produces short and low charged electron bunches, on the one hand to resolve the excitation transitions of atoms temporally by pump-probe electron diffraction experiments and on the other hand to investigate principal mechanisms of laser plasma acceleration. For both cases a high quality electron beam is required which can be identified with a small beam emittance. A standard magnet scan is used for the emittance measurement which is in case of a low charged bunch most sensitive to the beam size determination (RMS or $2^{\text {nd }}$ central moment of a distribution). Therefore the diagnostic and a routine to calculate proper central moments of an arbitrary distribution will be introduced and discussed.
\end{abstract}

Keywords: electron beam diagnostic, low charge application

\section{1. Introduction}

$2 \quad$ The Relativistic Electron Gun for Atomic Explo-

3 ration (REGAE, Fig. 1) at DESY is a small $5 \mathrm{MeV}$

4 linear accelerator with a bunch charge range of a

5 few to some hundreds fC. The beam energy is de- ${ }^{3}$

6 livered by an S-band photo-injector cavity. In addi-

7 tion to the gun a 4-cell buncher cavity is installed.

8 It is designed for ballistic bunching down to $10 \mathrm{fs}$.

9 Due to the low energy a beam optics consisting of

10 compact, symmetrically focusing solenoids is suffi-

cient.

12 The machine is built for two types of experi-

3 ments: first a time-resolved electron diffraction

14 experiment in order to make atomic transitions

15 'visible' [1, 2] and secondly investigations of new

16 plasma-wakefield acceleration schemes [3]. Both ex-

17 periments require a low transverse beam emittance

8 down to $10 \mathrm{~nm}$ (normalized emittance). Hence,

there are two challenges: generate such a high qual-

Email address: max.hachmann@desy.de (M. Hachmann) quality with high precision which is discussed below.

Due to the small energy spread as well as in first approximation negligible space charge effects a phase advance method can be utilized for the emittance measurement. For this purpose a charge sensitive detector system was developed which has the re- quired spatial resolution to measure the beam profile despite the unavoidable noise and background signals.

\section{Emittance measurement via a solenoid scan}

\subsection{Phase advance method}

A commonly used method to determine the transverse emittance of an electron bunch is a magnet scan. Here the phase advance between a magnet and a downstream screen is changed by varying the magnet current. Analyzing the RMS beam size as function of the focusing strength yields the emittance. Alternatively, it is possible to measure the beam size at different positions without any additional change of the optics. At REGAE the first method is used.

Assuming a small energy spread the trace space emittance is determined as

$$
\epsilon_{x}=\sqrt{\left\langle x^{2}\right\rangle\left\langle x^{\prime 2}\right\rangle-\left\langle x x^{\prime}\right\rangle^{2}}
$$

with $x$ as the transverse particle position and $x^{\prime}$ as the transverse angle relative to the longitudinal axis. The trace space emittance is related in the following way to the normalized emittance: $\epsilon_{x}=\frac{1}{\beta \gamma} \epsilon_{n, x}$ with $\beta=v / c$, the Lorentz factor $\gamma$, the velocity $v$ and the speed of light $c$. Furthermore, \langle\rangle denotes the central moment of a distribution, in this 


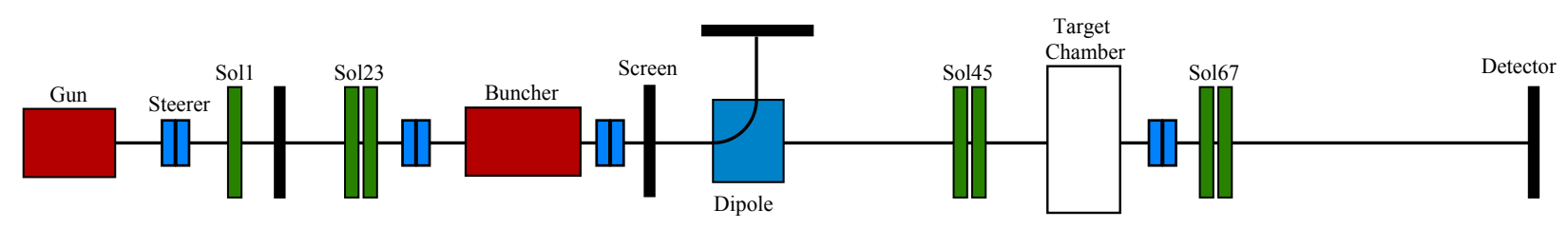

Figure 1: Schematic REGAE layout.

case the $2^{\text {nd }}$ central moment where the square root $\quad 85$ is normally called RMS: $\left\langle x^{2}\right\rangle=x_{r m s}^{2}$.

In order to measure the transverse beam emittance ${ }_{87}$ the envelope equation can be used [4]:

$$
x_{r m s}^{2}=\left(\begin{array}{llll}
R_{11}^{2} & 2 R_{11} R_{12} & R_{12}{ }^{2}
\end{array}\right)^{\top}\left(\begin{array}{l}
a_{1} \\
a_{2} \\
a_{3}
\end{array}\right)
$$

$$
\text { with } \begin{aligned}
a_{1} & =x_{0, r m s}{ }^{2} \\
a_{2} & =x_{0, r m s}\left(x_{0, r m s}\right)^{\prime} \\
a_{3} & =\frac{\epsilon_{x}^{2}}{x_{0, r m s}{ }^{2}}+\left(x_{0, r m s}\right)^{\prime 2} .
\end{aligned}
$$

$x_{r m s}$ denotes the RMS beam size at the screen, 99 $x_{0, r m s}$ the beam size and $\left(x_{0, r m s}\right)^{\prime}$ the envelope ${ }^{100}$ slope at the entrance of the solenoid. $\mathbf{R}$ denotes ${ }^{101}$ the transfer matrix between the solenoid and the ${ }^{\mathbf{1 0 2}}$ screen. In order to calculate the RMS beam size ${ }^{103}$ a Gaussian fit is often used. But the width of the ${ }^{104}$ normal distribution is only equal to the RMS if the ${ }^{\mathbf{1 0 5}}$ beam profile is really normal distributed. In any ${ }^{106}$ other case this assumption doesn't hold which fal- ${ }^{\mathbf{1 0 7}}$ sifies the results. It is important to emphasize that ${ }^{108}$ Eq. 2 holds for RMS quantities. But the calculation ${ }^{109}$ of the RMS is difficult because the pure beam sig- ${ }^{110}$ nal has to be separated from background and noise ${ }^{\mathbf{1 1 1}}$ signals which can not be avoided - just reduced. A post-processing routine for images will be introduced in the next section.

Taking Eq. 2 as a model describing the beam size development at a certain position in dependence of varying phase advances, the emittance can be found with the method of least-squares.

\subsection{Detector system at REGAE}

For the diffraction experiment at REGAE a highly sensitive detector system is installed which is capable of detecting single electrons. This detector combined with the solenoids Sol45 or Sol67 (Fig. 1) 112 can be used for the emittance measurements. The 113 detector contains a CsI-crystal-screen which is $\mathbf{1 1 4}$ grown onto light guides, called FOS (Fiber Optics 115
Scintillator) and was manufactured by Hamamatsu Photonics [5], and the Andor iXon Ultra 888 camera 6] with a charge sensitive Electron-Multiplying CCD (EMCCD). The pixel size of the CCD is $13 \mu \mathrm{m}$ and the size of each fiber of the FOS is $\sim 6 \mu \mathrm{m}$. 9o The overall spatial resolution of this detector sys1 tem is $\sim 16 \mu \mathrm{m}$ due to optics between scintillator and the EMCCD camera. For all presented measurements and results this resolution is sufficient. Due to the long drift section between the solenoids and the detector the smallest measured beam size is larger than $50 \mu \mathrm{m}$.

7 A schematic layout of the detector is shown in Fig. 2 The FOS is orientated perpendicular to the beam propagation. To avoid high energy photons or electrons hitting the EMCCD camera a mirror reflects the visible light emitted by the FOS under $90^{\circ}$ in direction of the camera. An Aluminum cover, directly layered onto the FOS reflects the emitted light of the FOS back in direction of the mirror. The whole setup is light-tight and the EMCCD is cooled down to $-70{ }^{\circ} \mathrm{C}$ in order to reduce the noise from stray light and electronics of the EMCCD, respectively. All these factors increase the charge sensitivity by a factor of $\sim 10^{3}$ compared to a 'standard scintillator-CCD-system' at REGAE ('Screen' in Fig. 1).

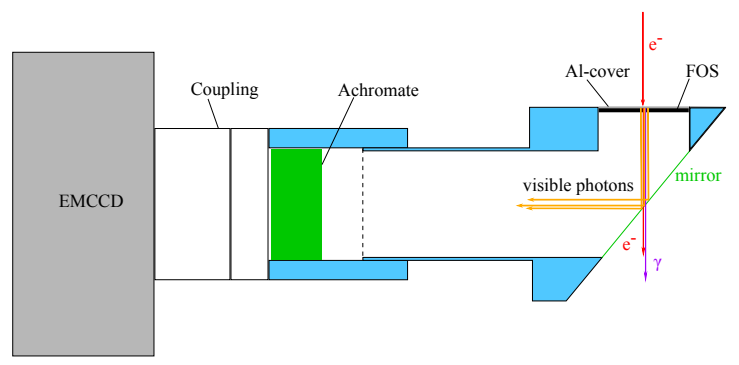

Figure 2: REGAE detector system layout.

In case of ultra-low bunch charges down to a few $\mathrm{fC}$ shot noise, the fluctuation of the number of electrons from shot-to-shot, has a big contribution to the signal-to-noise ratio of the actual detector sys- 
tem at REGAE even if it is not the only significant 158 factor. A new detector system is in the planning 159 stage and should be sensitive enough that the shot 160 noise is the limiting factor of this system. The new ${ }_{161}$ system will hit the limits of achievable sensitivity, 162 but will improve the measurements for all kinds of experiments as well as diagnostic issues.

\section{Image post-processing}

Compared to a more sophisticated image postprocessing routine we developed to deal for example with the shot noise as the main source of background signal [7, we are currently facing a broad and distinct halo which can be clearly seen with our detector system. An example is shown in Fig. 3 Investigations of the origin of the halo are still ongoing. Till now it could not be clarified whether it is electron beam related or an artifact of the detector system. Using collimators, which are cutting out a round part of the electron beam, is changing the proportion of the peaked signal and the halo but still the ratio of the halo is significant $(>15 \%)$ and cannot be ignored.

But nevertheless the effect of this halo on e.g. a diffraction experiment is small. A distinct image of a diffraction pattern is mainly depending on the geometry of the imaging system and the probe's quality - in this case on the geometry and beam parameters of the REGAE accelerator. Therefore the peaked part of the beam with a higher beam quality (or smaller emittance) has the largest influence ${ }_{168}$ on the imaging of a diffraction pattern. As a conse- 169 quence we decided to apply an intensity cut before 170 using recorded data for further evaluations. The ${ }_{171}$ halo as well as the 'peak on top' can be described ${ }_{172}$ by the superposition of two Gaussian distributions: ${ }_{173}$

$$
\begin{aligned}
f(x)= & a_{1} \exp \left(-\frac{\left(x-\mu_{1}\right)^{2}}{2 \sigma_{1}^{2}}\right) \\
& +a_{2} \exp \left(-\frac{\left(x-\mu_{2}\right)^{2}}{2 \sigma_{2}^{2}}\right) .
\end{aligned}
$$

$a_{i}$ is the distribution's amplitude, $\mu_{i}$ describes 181 the barycenter and $\sigma_{i}$ the standard deviation (or 182 $2^{\text {nd }}$ central moment) of each distribution with 183 $i=1,2$. The aim is to use only the peaked Gaussian profile ${ }_{185}$ for the described emittance measurement method ${ }_{186}$ (Sec. 2.1). Eq. 3 is used to fit the profiles of the 187 focused beam to determine the cut-intensity in order to use it for the whole image set at a certain magnet current. The cut-intensity is defined as the integrated intensity of the first Gaussian distribution:

$$
I_{\text {cut }}=\int_{\infty}^{\infty} a_{1} \exp \left(-\frac{\left(x-\mu_{1}\right)^{2}}{2 \sigma_{1}^{2}}\right) \mathrm{d} x,
$$

assuming $a_{1}, \mu_{1}$ and $\sigma_{1}$ are the parameters of the peaked Gaussian distribution. From the cut off profiles the needed $2^{\text {nd }}$ central moments can be calculated and used for the emittance fit routine.

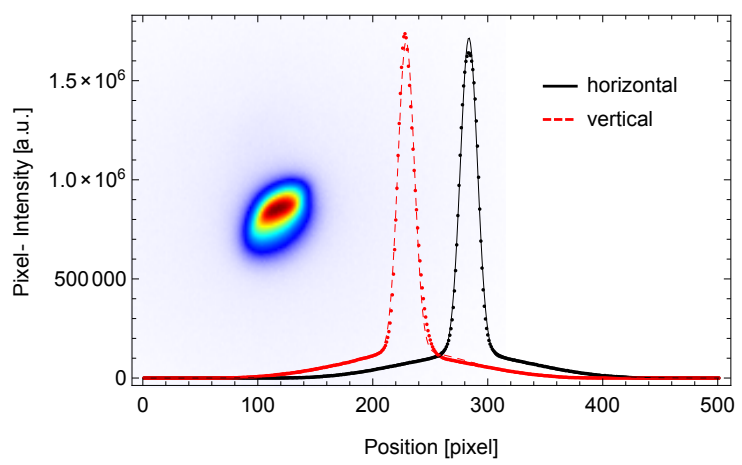

Figure 3: Measured beam profiles at REGAE - projections and 2D-colored profile. Double Gaussian fits (solid lines) were applied.

\section{Measurements}

Due to a repaired vacuum leak of the gun cavity only moderate field gradients up to $60 \mathrm{MV} / \mathrm{m}$ can be set presently. The cavity will be replaced but for now it limits the mean beam energy and the contributions of space charge effects to the emittance growth inside the gun cavity as well as during the beam transport are larger. An exemplary emittance measurement is shown in Fig. 4 The measured mean beam energy was $E_{k i n}=2.45 \mathrm{MeV}$. The bunch charge was determined as $Q_{\text {bunch }}=37.6(6) \mathrm{fC}$ - the number in brackets denotes the uncertainty of the last digit. The least-square fit parameters are shown in Tab. 1 (calculated following [8]). The goodness of the fit is estimated by the reduced Chi Square $\tilde{\chi}^{2}$ which should tend to one.

Another interesting concept which was investigated is the usage of collimators and the dependence on the emittance. With different diameters different amounts of charge can be cut out. A 


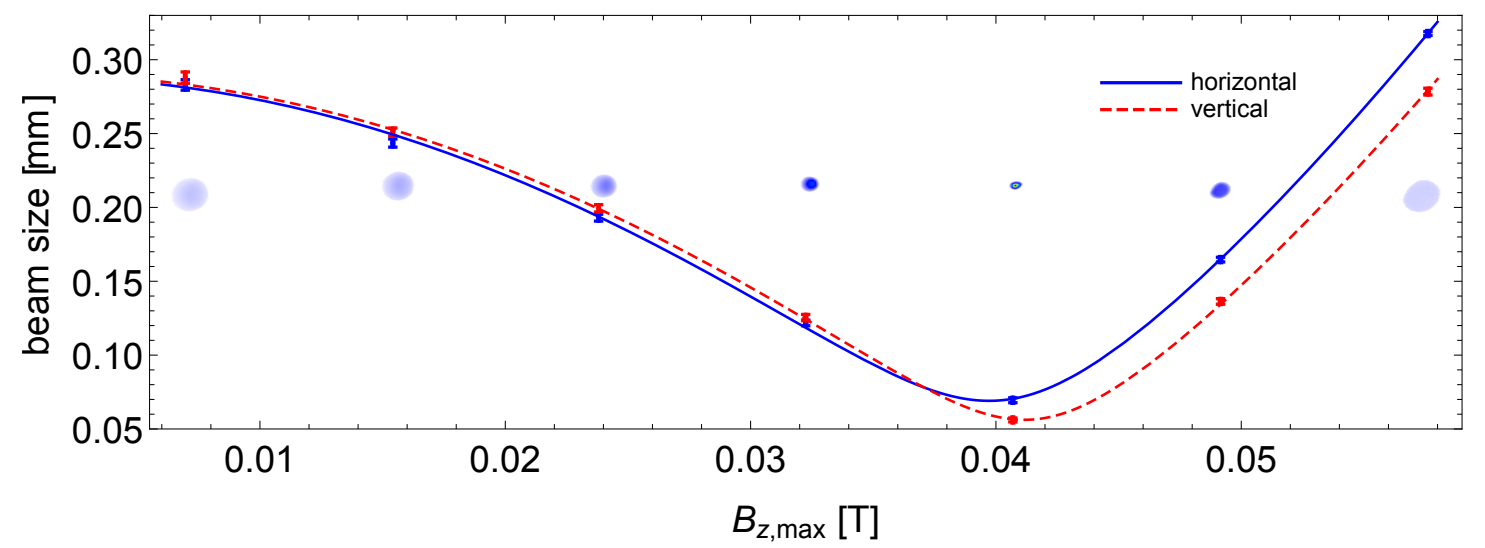

Figure 4: Measured beam size at the detector (Fig. 1) in dependence of the maximum magnetic field $B_{z, m a x}$ of solenoid $S o l 67$. Solid lines represent least-square fits which were done with the introduced model of Eq. 2. The 2D-beam profile of each magnet setting is depicted in the background.

Table 1: Least-square fit parameters and uncertainties of a solenoid scan at REGAE

\begin{tabular}{ccc}
\hline & horizontal & vertical \\
\hline$\epsilon_{\mathbf{n}}[\pi \mathrm{mm} \mathrm{mrad}]$ & $0.0271(7)$ & $0.0209(8)$ \\
$\mathbf{x}_{\mathbf{0}, \mathbf{r m s}}[\mathrm{mm}]$ & $0.244(1)$ & $0.231(1)$ \\
$\left(\mathbf{x}_{\mathbf{0}, \mathbf{r m s}}\right)^{\prime}[\mu \mathrm{\mu ad}]$ & $12.8(3)$ & $17.7(3)$ \\
$\tilde{\chi}^{\mathbf{2}}$ & 2.7 & 1.2 \\
\hline
\end{tabular}

reduced beam size reduces the emittance as well (Eq. 1). Due to the collimators an even smaller norm. trans. emittances could be achieved and measured. An emittance measurement for different collimator diameters is shown in Fig. 5 . At the same time as the charge is reduced of course the 208 space charge effects are reduced as well. Hence, the 209 reduced emittance is not a pure effect of the beam $2 \mathbf{2 1 0}$ size reduction.

\section{Conclusion and Outlook}

Ultra-low emittance measurements for ultra-low ${ }^{215}$ bunch charges could be successfully performed at ${ }_{217}$ REGAE. The limit of the introduced diagnostic method is showing up at the transition of signal to noise where a loss of information is unavoidable and cannot be regained. This loss could be minimized by the introduced detector system. In order to improve the sensitivity a new detector system will be setup at REGAE in the next months. The investigations of the observed halo are still ongoing.

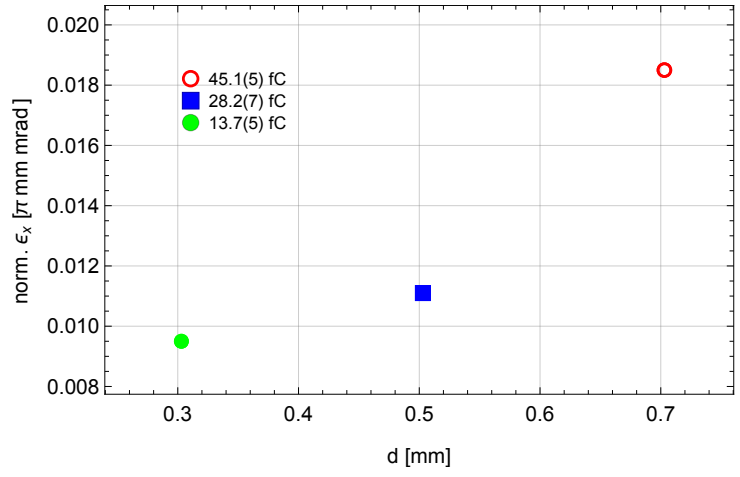

Figure 5: The norm. transverse emittance in dependence of the collimator diameter.

Because always some relevant beam signal gets cut every introduced cut potentially falsify the results. Solving this issue offers the possibility to measure and determine the 'uncut-emittances' of the REGAE electron beam.

With higher mean beam energies space charge effects can be suppressed and more charge can be accelerated while the emittance is still conserved. The replacement of the REGAE gun cavity will help to achieve this and normalized tranverse emittances down to $10^{-3} \mathrm{~mm}$ mrad at a few $\mathrm{fC}$ seem to be feasible. 


\section{References}

[1] Jason R. Dwyer, Christoph T. Hebeisen, Ralph Ernstorfer, Maher Harb, Vatche B. Deyirmenjian, Robert E. Jordan, and R.J. Dwayne Miller. Femtosecond electron diffraction: 'Making the molecular movie'. Phil. Trans. R. Soc. A, 364:741-778, January 2006.

[2] Stephanie Manz, Albert Casandruc, Dongfang Zhang, Yinpeng Zhong, Rolf A. Loch, Alexander Marx, Taisuke Hasegawa, Lai Chung Liu, Shima Bayesteh, Hossein Delsim-Hashemi, Matthias Hoffmann, Matthias Felber, Max Hachmann, Frank Mayet, Julian Hirscht, Sercan Keskin, Masaki Hada, Sascha W. Epp, Klaus Floettmann, and R.J. Dwayne Miller. Mapping atomic motions with ultrabright electrons: Towards fundamental limits in space-time resolution. Faraday Discuss., 177:476-491, January 2015.

[3] Benno Zeitler, Irene Dornmair, Tim Gehrke, Mikheil Titberidze, Andreas R. Maier, Bernhard Hidding, Klaus Floettmann, and Florian Gruener. Merging conventional and laser wakefield accelerators. In Proc. SPIE, volume 8779, 2013.

[4] Max Hachmann. Transverse emittance measurement at REGAE via a solenoid scan. Master's thesis, University of Hamburg, Germany, 2013.

[5] Hamamatsu Photonics. X-ray scintillator FOS ACS ALS GPXS ALS. http://www.hamamatsu.

com/resources/pdf/etd/FOS_ACS_GPXS_ALS_

2016. Online; accessed 07-January-2016.

[6] Andor Technology Ltd. Andor iXon3 EMCCD.

http://www . andor.com/pdfs/literature/Andor_

iXon3_EMCCD_Brochure.pdf, 2016. Online; accessed 07-January-2016.

[7] Max Hachmann and Klaus Floettmann. Transverse emittance measurement at REGAE. In Proc. IPAC, 2015.

[8] M. G. Minty and F. Zimmermann. Measurement and Control of Charged Particle Beams. Springer-Verlag, Berlin Heidelberg, 2003. 